\title{
Evaluation of effectiveness of granulocyte-macrophage colony-stimulating factor therapy to cancer patients after chemotherapy: a meta-analysis
}

\author{
Wen-Liang Yu ${ }^{1}$ and Zi-Chun Hua ${ }^{1,2,3}$ \\ ${ }^{1}$ The State Key Laboratory of Quality Research in Chinese Medicine, Macau Institute for Applied Research in Medicine and \\ Health, Macau University of Science and Technology, Macao, China \\ ${ }^{2}$ The State Key Laboratory of Pharmaceutical Biotechnology, School of Life Sciences, Nanjing University, Nanjing, China \\ ${ }^{3}$ Changzhou High-Tech Research Institute of Nanjing University and Jiangsu TargetPharma Laboratories Inc., Changzhou, \\ China \\ Correspondence to: Zi-Chun Hua, email: huazc@nju.edu.cn \\ Keywords: GM-CSF; hematologic index; meta-analysis \\ Received: August 14, 2017 Accepted: February 28, 2018 Published: June 15, 2018 \\ Copyright: Yu et al. This is an open-access article distributed under the terms of the Creative Commons Attribution License 3.0 \\ (CC BY 3.0), which permits unrestricted use, distribution, and reproduction in any medium, provided the original author and source \\ are credited.
}

\section{ABSTRACT}

The impact of granulocyte-macrophage colony stimulating factor (GM-CSF) on hematologic indexes and complications remains existing contradictory evidence in cancer patients after treatment of chemotherapy. Eligible studies up to March 2017 were searched and reviewed from PubMed and Wanfang databases. Totally 1043 cancer patients from 15 studies were included in our research. The result indicated that GM-CSF could significantly improve white blood cells count (SMD $=1.16,95 \%$ CI: $0.71-1.61, Z=5.03, P<0.00001$ ) and reduce the time to leukopenia recovery (SMD $=-0.85,95 \% \mathrm{CI}:-1.16--0.54, Z=5.38, P<0.00001)$ in cancer patients after treatment of chemotherapy. It also could improve absolute neutrophil count (SMD = $1.11,95 \% \mathrm{CI}: 0.39-1.82, Z=3.04, P=0.002$ ) and significantly shorten the time to neutropenia recovery (SMD $=-1.47,95 \% \mathrm{CI}$ : $-2.20--1.75, Z=3.99, P<0.0001)$. However, GM-CSF could not improve blood platelet (SMD $=0.46,95 \%$ CI: -0.37 $-1.29, Z=1.10, P=0.27)$. And GM-CSF had significant connection with fever ( $R R=$ 3.44, 95\% CI: $1.43-8.28, Z=2.76, P=0.006)$. The publication bias existed in the data of the impact of GM-CSF on blood platelet and complication. In conclusions, GMCSF had an intimate association with some hematologic indexes and complications. Our study suggested that more hematological indexes and even more other indexes need to be observed in future studies.

\section{INTRODUCTION}

Chemotherapy has been widely used for treating different cancers for many years. Whereas chemotherapy can lead to many adverse drug reactions, such as hematologic toxicity, thromboembolism, and neurotoxicity. [1-3]. Hematologic toxicity is a common adverse reaction such as neutropenia and leukopenia. It not only delays the time of next therapy but also leads to the life-threatening events (such as severe infection, bleeding, copper deficiency and protein malnutrition) if decreased blood cells had not been managed timely $[4,5]$. The previous studies had confirmed that chemotherapyinduced neutropenia or leukopenia could reduce survival rates in patients with advanced cancer $[3,6]$. Thus, the hematologic indexes, including white blood cells (WBC) count, absolute neutrophil count (ANC), blood platelet (PLT) count and monocytes count, are important objective indexes in cancer patients after chemotherapy.

GM-CSF is the cytokine most extensively used as hemopoietin in the clinical practice, it can promote the activation, proliferation, and differentiation of myeloid 
precursor cells in the body. Beside GM-CSF plays an important role in the recruitment, development, and maturation of dendritic cells, which are necessary for the subsequent $\mathrm{T}$ helper cell type 1 and cytotoxic T lymphocyte activation. GM-CSF also can enhance the function of mature granulocytes and mononuclear phagocytes [5, 7-9]. In the 1990s, GM-CSF was approved by Food and Drug Administration (FDA) as a drug for treatment of older adults with acute myeloid leukemia, after induction chemotherapy, to shorten the time to neutrophil recovery and to reduce the incidence of life-threatening infections. Thus GM-CSF is often used to treat chemotherapy-induced neutropenia and leucopenia [8, 10]. GM-CSF was also approved by FDA for myeloid reconstitution following allogeneic bone marrow transplantation (BMT), autologous BMT or peripheral blood stem cell transplantation. Furthermore, GM-CSF was approved for peripheral blood stem cell mobilization, BMT failure, and engraftment delay. GM-CSF also has many proinflammatory functions [11]. The trade name of GM-CSF includes sargramostim, leucomax (American name), "Te Li Er", "Ge Ning" (Chinese name), and molgramostim (Indian name).

GM-CSF has been used to treat chemotherapyincluded neutropenia and leucopenia for many years, and many different clinical responses and data are accumulated. However, these clinical data and responses, including the impact of GM-CSF on WBC count, ANC, BLT, and complications, still lack systematic analysis and evaluation. Hence this study aims to quantify the data from previous clinical studies via meta-analysis, for systematically analyzing and evaluating the clinical impact of GM-CSF on hematologic indexes and complications.

\section{RESULTS}

\section{Research results and quality assessment}

The process of the literature search was included in a PRISMA flow diagram (Figure 1). The preliminary screening identified 2449 potentially relevant publications by reviewing titles and abstracts, including 176 duplicates, 935 non-research article, and 1293 irrelevant studies. And then we screened the full-texts of the remaining 45 articles, 28 and 2 papers were excluded with insufficient result data and radiotherapy, respectively. Finally, 15 studies [12-26] (Table 1) were included in our research, including 5 English papers [12-16] and 10 Chinese papers [17-26]. The quality assessment of 15 studies was shown in Table 2. The agreement for selection of studies between two authors was high.

\section{Impact of GM-CSF on WBC count in cancer patients}

Eight studies [17, 18, 20-24, 26] which included 469 cancer patients after chemotherapy reported the association between GM-CSF and WBC count. Homogeneity test showed that these studies had not homogeneity $\left(\chi^{2}=\right.$ $66.05, P<0.00001, \mathrm{I}^{2}=89 \%$, Figure 2A). Thus we used the random-effect model to make statistics. And the result demonstrated that GM-CSF was associated with increased WBC count $(\mathrm{SMD}=1.16,95 \% \mathrm{CI}$ : $0.71-1.61$, $\mathrm{Z}=5.03, P<0.00001$, Figure 2A). Furthermore, we analyzed the connections between GM-CSF and the time of leukopenia. Five studies [18, 20, 21, 23, 26], included 281 patients, showed the data regarding the effect of GM-CSF on the time to neutropenia. And these studies had not homogeneity as well $\left(\chi^{2}=12.76, P=0.01, \mathrm{I}^{2}=\right.$ $69 \%$, Figure 2B). Thus, the data were calculated by the random-effect model. The result demonstrated that GMCSF shortened the time to WBC count recovery (SMD $=-0.85,95 \% \mathrm{CI}:-1.16--0.54, \mathrm{Z}=5.38, P<0.00001$, Figure 2B), thus GM-CSF could made the WBC count recovery more quickly.

\section{Impact of GM-CSF on ANC in cancer patients}

Eight studies [13, 16, 18, 21-24, 26] provided the data regarding $\mathrm{ANC}$, which included 533 cancer patients after chemotherapy. All studies had significant heterogeneity $\left(\chi^{2}=176.48, P<0.00001, \mathrm{I}^{2}=96 \%\right.$, Figure 3A). Thus, random-effect model was used to calculate them, and the result suggested that GM-CSF was connected with increased ANC (SMD = 1.11, 95\% CI: $0.39-1.82, \mathrm{Z}=3.04, P=0.002$, Figure $3 \mathrm{~A})$. And then, five studies $[13,18,21,23,26]$ reported the data concerning the time to neutropenia recovery, which included 246 patients. Homogeneity test revealed not homogeneity $\left(\chi^{2}=49.52, P<0.00001, \mathrm{I}^{2}=92 \%\right.$, Figure $3 \mathrm{~B})$, these data were calculated by using random-effect model. The result confirmed that GM-CSF could shorten the time to ANC recovery $(\mathrm{SMD}=-1.47,95 \% \mathrm{CI}:-2.20-$ $-1.75, \mathrm{Z}=3.99, P<0.0001$, Figure 3B).

\section{Impact of GM-CSF on PLT count in cancer patients}

Six studies $[13,16,18,24-26]$ including 375 cancer patients after chemotherapy, offered the data concerning PLT count. Homogeneity test showed all studies were heterogeneous $\left(\chi^{2}=133.53, P<0.00001, \mathrm{I}^{2}=96 \%\right.$, Figure $4)$. Thus we used random-effect model to calculate them. The result showed that the association between GM-CSF and PLT count does not exist $(\mathrm{SMD}=0.46,95 \% \mathrm{CI}:-0.37$ $-1.29, \mathrm{Z}=1.10, P=0.27$, Figure 4).

\section{Complications}

Because of individual differences, different patients often had different degrees of complications after treatment with GM-CSF, such as fever, local reactions, bone and muscle pain. Except for fever, most of the 
complications could be tolerated and do not lead to serious consequences. Ten studies [12, 14-19, 21, 23, 25, 26] provide the data of fever, and 805 patients were included.
Homogeneity test showed that there is no homogeneity in these studies $\left(\chi^{2}=34.98, P<0.0001, \mathrm{I}^{2}=74 \%\right.$, Figure 5). Hence random-effect model was used to calculate these

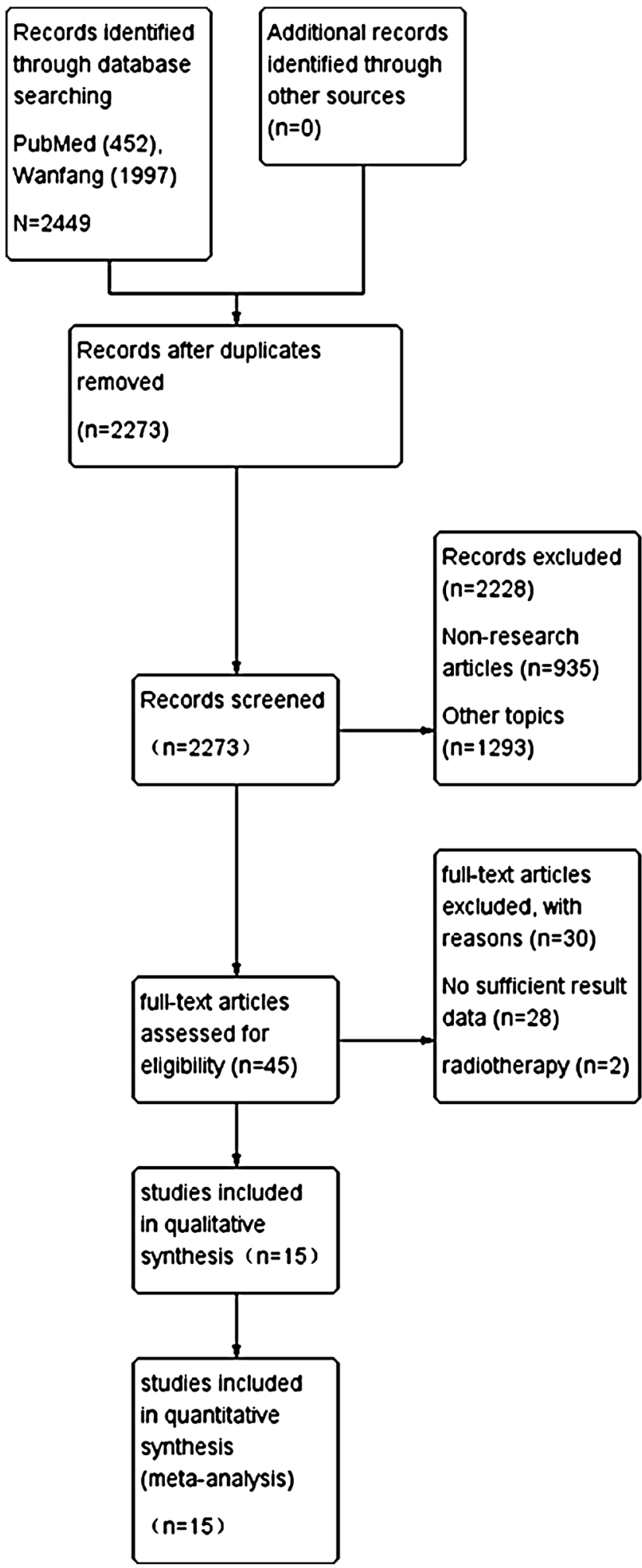

Figure 1: Flow-diagram of the literature selection process. 
Table 1: Basic characteristics of studies included in this meta-analysis

\begin{tabular}{|c|c|c|c|c|c|c|c|c|c|c|c|c|}
\hline $\begin{array}{l}\text { Study } \\
\text { publish year }\end{array}$ & $\begin{array}{l}\text { Country/ } \\
\text { race }\end{array}$ & $\begin{array}{l}\text { Type } \\
\text { of } \\
\text { study }\end{array}$ & $\begin{array}{c}\text { Treatment } \\
\text { details }\end{array}$ & $\begin{array}{l}\text { No. of } \\
\text { patient }\end{array}$ & $\begin{array}{c}\text { Sex } \\
\text { ratio } \\
(\text { male } \%)\end{array}$ & Age & $\begin{array}{c}\text { Weight } \\
\text { (kg) }\end{array}$ & KPS & $\begin{array}{l}\text { Sources of } \\
\text { medication }\end{array}$ & $\begin{array}{c}\text { Dosage and } \\
\text { duration of } \\
\text { GM-CSF }\end{array}$ & $\begin{array}{c}\text { Clinical } \\
\text { response }\end{array}$ & $\begin{array}{l}\text { Toxic } \\
\text { and } \\
\text { side } \\
\text { effects }\end{array}$ \\
\hline $\begin{array}{l}\text { B Kopf et al. } \\
2006\end{array}$ & $\begin{array}{l}\text { Italy/ } \\
\text { Europe }\end{array}$ & $\begin{array}{c}\text { clinical } \\
\text { trial }\end{array}$ & CT & 29 & 48 & $21-56$ & - & - & - & $\begin{array}{l}1 \text { time per } 1 \mathrm{~d} \\
(5 \mathrm{mg} / \mathrm{kg})\end{array}$ & $87.5 \%$ & Y \\
\hline $\begin{array}{l}\text { Nathan L et } \\
\text { al. } 1999\end{array}$ & $\begin{array}{l}\text { Canada/ } \\
\text { North } \\
\text { America }\end{array}$ & $\begin{array}{c}\text { clinical } \\
\text { trial }\end{array}$ & $\mathrm{CT}$ & 20 & 0 & $31-69$ & - & - & America & $\begin{array}{l}1 \text { time per } 1 \mathrm{~d} \\
\left(250 \mu \mathrm{g} / \mathrm{m}^{2}\right)\end{array}$ & $82.9 \%$ & $\mathrm{~N}$ \\
\hline $\begin{array}{l}\text { Montero et } \\
\text { al. } 2005\end{array}$ & $\begin{array}{l}\text { America/ } \\
\text { North } \\
\text { America }\end{array}$ & $\begin{array}{c}\text { clinical } \\
\text { trial }\end{array}$ & $\mathrm{CT}$ & 23 & - & $31-69$ & - & $70-90$ & America & $\begin{array}{l}1 \text { time per 1d } \\
\left(250 \mu \mathrm{g} / \mathrm{m}^{2}\right)\end{array}$ & $96 \%$ & Y \\
\hline $\begin{array}{l}\text { Stephen E et } \\
\text { al. } 1996\end{array}$ & $\begin{array}{l}\text { America/ } \\
\text { North } \\
\text { America }\end{array}$ & $\begin{array}{c}\text { clinical } \\
\text { trial }\end{array}$ & $\mathrm{CT}$ & 142 & 0 & $25-69$ & $46-133$ & $\geq 80$ & America & $\begin{array}{l}1 \text { time per } 1 \mathrm{~d} \\
\left(250 \mu \mathrm{g} / \mathrm{m}^{2}\right)\end{array}$ & $97 \%$ & Y \\
\hline $\begin{array}{l}\text { A. Le Cesne } \\
\text { et al. } 2000\end{array}$ & $\begin{array}{l}\text { France/ } \\
\text { Europe }\end{array}$ & $\begin{array}{c}\text { clinical } \\
\text { trial }\end{array}$ & $\mathrm{CT}$ & 294 & 42 & $19-76$ & - & $\geq 70$ & Germany & $\begin{array}{l}1 \text { time per } 1 \mathrm{~d} \\
\left(250 \mu \mathrm{g} / \mathrm{m}^{2}\right)\end{array}$ & $21.3 \%$ & Y \\
\hline $\begin{array}{l}\text { He et al. } \\
2001\end{array}$ & $\begin{array}{l}\text { China/ } \\
\text { Asia }\end{array}$ & $\begin{array}{c}\text { clinical } \\
\text { trial }\end{array}$ & $\mathrm{CT}$ & 50 & 64 & $8-77$ & - & - & China & $\begin{array}{l}1 \text { time per } 1 \mathrm{~d} \\
(75 \mu \mathrm{g} / \mathrm{d})\end{array}$ & $92 \%$ & Y \\
\hline $\begin{array}{l}\text { Zhou et al. } \\
1999\end{array}$ & $\begin{array}{l}\text { China/ } \\
\text { Asia }\end{array}$ & $\begin{array}{c}\text { clinical } \\
\text { trial }\end{array}$ & $\mathrm{CT}$ & 60 & 63 & $16-68$ & $40-80$ & - & China & $\begin{array}{l}1 \text { time per } 1 \mathrm{~d} \\
(5 \mu \mathrm{g} / \mathrm{kg})\end{array}$ & $86.7 \%$ & Y \\
\hline Sun 2005 & $\begin{array}{l}\text { China/ } \\
\text { Asia }\end{array}$ & $\begin{array}{c}\text { clinical } \\
\text { trial }\end{array}$ & $\mathrm{CT}$ & 28 & 71 & $24-71$ & - & $\geq 60$ & China & $\begin{array}{l}1 \text { time per } 1 \mathrm{~d} \\
(150 \mu \mathrm{g} / \mathrm{d})\end{array}$ & $92.9 \%$ & Y \\
\hline $\begin{array}{l}\text { Liu et al. } \\
2000\end{array}$ & $\begin{array}{l}\text { China/ } \\
\text { Asia }\end{array}$ & $\begin{array}{c}\text { clinical } \\
\text { trial }\end{array}$ & $\mathrm{CT}$ & 55 & 60 & $14-70$ & - & $\geq 60$ & China & $\begin{array}{l}1 \text { time per } 1 \mathrm{~d} \\
(5 \mu \mathrm{g} / \mathrm{kg})\end{array}$ & $73.7 \%$ & $\mathrm{~N}$ \\
\hline $\begin{array}{l}\text { Chen et al. } \\
2003\end{array}$ & $\begin{array}{l}\text { China/ } \\
\text { Asia }\end{array}$ & $\begin{array}{c}\text { clinical } \\
\text { trial }\end{array}$ & $\mathrm{CT}$ & 50 & 58 & $23-71$ & - & $>60$ & China & $\begin{array}{l}1 \text { time per } 1 \mathrm{~d} \\
(150 \mu \mathrm{g} / \mathrm{d})\end{array}$ & - & Y \\
\hline $\begin{array}{l}\text { Liu et al. } \\
2000\end{array}$ & $\begin{array}{l}\text { China/ } \\
\text { Asia }\end{array}$ & $\begin{array}{c}\text { clinical } \\
\text { trial }\end{array}$ & $\mathrm{CT}$ & 78 & 54 & $23-78$ & - & $>60$ & China & $\begin{array}{l}1 \text { time per } 1 \mathrm{~d} \\
(75 \mu \mathrm{g} / \mathrm{d})\end{array}$ & - & Y \\
\hline $\begin{array}{l}\text { Zhou et al. } \\
1999\end{array}$ & $\begin{array}{l}\text { China/ } \\
\text { Asia }\end{array}$ & $\begin{array}{c}\text { clinical } \\
\text { trial }\end{array}$ & $\mathrm{CT}$ & 60 & 60 & $16-70$ & $40-95$ & $70-100$ & China & $\begin{array}{l}1 \text { time per } 1 \mathrm{~d} \\
(5 \mu \mathrm{g} / \mathrm{kg})\end{array}$ & - & Y \\
\hline Ji 2015 & $\begin{array}{l}\text { China/ } \\
\text { Asia }\end{array}$ & $\begin{array}{c}\text { clinical } \\
\text { trial }\end{array}$ & CT & 60 & 50 & $25-68$ & - & - & China & $\begin{array}{l}1 \text { time per } 1 \mathrm{~d} \\
(100 \mu \mathrm{g} / \mathrm{d})\end{array}$ & - & $\mathrm{N}$ \\
\hline $\begin{array}{l}\text { Yuan et al. } \\
2002\end{array}$ & $\begin{array}{l}\text { China/ } \\
\text { Asia }\end{array}$ & $\begin{array}{c}\text { clinical } \\
\text { trial }\end{array}$ & $\mathrm{CT}$ & 30 & 67 & $10-69$ & - & - & China & $\begin{array}{l}1 \text { time per } 1 \mathrm{~d} \\
(3-5 \mu \mathrm{g} / \mathrm{kg})\end{array}$ & $93.33 \%$ & Y \\
\hline $\begin{array}{l}\text { Zhou et al. } \\
1999\end{array}$ & $\begin{array}{l}\text { China/ } \\
\text { Asia }\end{array}$ & $\begin{array}{c}\text { clinical } \\
\text { trial }\end{array}$ & $\mathrm{CT}$ & 56 & 60 & $17-67$ & $45-84$ & - & China & $\begin{array}{l}1 \text { time per } 1 \mathrm{~d} \\
(5 \mu \mathrm{g} / \mathrm{kg})\end{array}$ & - & Y \\
\hline
\end{tabular}

Notes: CT: chemotherapy. KPS: Karnofsky Performance Status. Y: the article mentions toxic and side effects. N: the article do not have or do not mention toxic and side effects.

data. The results suggested that GM-CSF had significant connection with fever $(\mathrm{RR}=3.44,95 \% \mathrm{CI}: 1.43-8.28, \mathrm{Z}$ $=2.76, P=0.006$, Figure 5).

\section{Publication bias}

As shown in Figure 6, the funnel plots for evaluating the publication bias for the impact of GMCSF on hematologic index and complications. Open circles represent studies included in the meta-analysis. The perpendicular in the center indicates the summary proportion. And the other two dotted lines represent the
95\% CI. On the visual assessment of funnel plot, there is no evidence of publication bias was revealed on the association between GM-CSF with WBC count, the time to neutropenia recovery and leucopenia recovery (Figure $6 \mathrm{~A}, 6 \mathrm{~B}$ and 6D). However, the publication bias existed on the studies regarding the impact of GM-CSF on ANC, PLT count, and complications.

\section{DISCUSSION}

Recent studies regarding chemotherapeutic agents show that targeted therapies have received 
Table 2: Reporting quality of 15 randomized control trials based on the CONSORT 2010 Checklist [n (\%)]

\begin{tabular}{|c|c|c|c|c|}
\hline Section/topic & No & Checklist item & $\mathbf{1}^{\mathrm{a}}$ & $\mathbf{0}^{\mathbf{b}}$ \\
\hline \multicolumn{5}{|c|}{ Title and abstract } \\
\hline & $1 \mathrm{a}$ & $\begin{array}{l}\text { Identification as a } \\
\text { randomized trial in } \\
\text { the title }\end{array}$ & $\begin{array}{c}5 \\
(33.3)\end{array}$ & $\begin{array}{c}10 \\
(66.7)\end{array}$ \\
\hline & $1 b$ & $\begin{array}{l}\text { Structured summary } \\
\text { of trial design, } \\
\text { methods, results, } \\
\text { and conclusions (for } \\
\text { specific guidance } \\
\text { see CONSORT for } \\
\text { abstracts) }\end{array}$ & $\begin{array}{c}10 \\
(66.7)\end{array}$ & $\begin{array}{c}5 \\
(33.3)\end{array}$ \\
\hline
\end{tabular}

\section{Introduction}

Background and objectives

Scientific background

2a and explanation of

$8(53.3) \quad 2(13.3) \quad 5(33.3)$

rationale

$2 \mathrm{~b}$

Specific objectives or hypotheses

$\begin{array}{ccc}10 & 3 & 1 \\ (66.7) & (20.0) & (6.7)\end{array}$

\section{Methods}

Trial design

$\begin{array}{ccc}\text { Description of trial } & & \\ \text { design (such as } & 15 & 0 \\ \text { 3a } \quad \text { parallel, factorial) } & (100) & (0.0) \\ \text { including allocation } & & \\ \quad \text { ratio }\end{array}$

Important changes to methods after trial 3b commencement (such as eligibility criteria),

$\begin{array}{cc}0 & 0 \\ (0.0) & (0.0)\end{array}$

unable to judge with reasons

$4 a$

Eligibility criteria for participants

$\begin{array}{ccc}13 & 0 & 2 \\ (86.7) & (0.0) & (13.3)\end{array}$

Participants

Interventions

Settings and locations $4 \mathrm{~b}$ where the data were collected

$\begin{array}{cc}2 & 13 \\ (13.3) & (86.7)\end{array}$

The interventions for each group with sufficient details to 5 allow replication, including how and

(100) $\quad(0.0)$

when they were actually administered

(continued) 


\begin{tabular}{|c|c|c|c|c|c|c|}
\hline Section/topic & No & Checklist item & $1^{a}$ & $\mathbf{0}^{\mathrm{b}}$ & $\mathbf{N I}^{\mathrm{c}}$ & Remark \\
\hline \multirow[t]{2}{*}{ Outcomes } & $6 \mathrm{a}$ & $\begin{array}{l}\text { Completely defined } \\
\text { prespecified primary } \\
\text { and secondary } \\
\text { outcome measures, } \\
\text { including how and } \\
\text { when they were } \\
\text { assessed }\end{array}$ & $\begin{array}{c}0 \\
(0.0)\end{array}$ & $\begin{array}{c}15 \\
(100)\end{array}$ & & \\
\hline & $6 b$ & $\begin{array}{l}\text { Any changes to trial } \\
\text { outcomes after the } \\
\text { trial commenced, } \\
\text { with reasons }\end{array}$ & $\begin{array}{c}3 \\
(20.0)\end{array}$ & $\begin{array}{c}12 \\
(80.0)\end{array}$ & & \\
\hline \multirow[b]{2}{*}{ Sample size } & $7 \mathrm{a}$ & $\begin{array}{l}\text { How sample size was } \\
\text { determined }\end{array}$ & $\begin{array}{c}14 \\
(93.3)\end{array}$ & $\begin{array}{c}1 \\
(6.7)\end{array}$ & & \\
\hline & $7 \mathrm{~b}$ & $\begin{array}{l}\text { When applicable, } \\
\text { explanation of any } \\
\text { interim analyses and } \\
\text { stopping guidelines }\end{array}$ & $\begin{array}{c}5 \\
(33.3)\end{array}$ & $\begin{array}{c}10 \\
(66.7)\end{array}$ & & \\
\hline \multirow[b]{2}{*}{ Sequence generation } & $8 \mathrm{a}$ & $\begin{array}{l}\text { Method used to } \\
\text { generate the random } \\
\text { allocation sequence }\end{array}$ & $9(60.0)$ & $6(40.0)$ & & \\
\hline & $8 b$ & $\begin{array}{c}\text { Type of } \\
\text { randomization; details } \\
\text { of any restriction } \\
\text { (such as blocking and } \\
\text { block size) }\end{array}$ & $8(53.3)$ & $7(46.7)$ & & \\
\hline $\begin{array}{l}\text { Allocation concealment } \\
\text { mechanism }\end{array}$ & 9 & $\begin{array}{l}\text { Mechanism used } \\
\text { to implement the } \\
\text { random allocation } \\
\text { sequence (such } \\
\text { as sequentially } \\
\text { numbered containers), } \\
\text { describing any steps } \\
\text { taken to conceal } \\
\text { the sequence until } \\
\text { interventions were } \\
\text { assigned }\end{array}$ & $7(46.7)$ & $8(53.3)$ & & \\
\hline Implementation & 10 & $\begin{array}{l}\text { Who generated the } \\
\text { random allocation } \\
\text { sequence, who } \\
\text { enrolled participants, } \\
\text { and who assigned } \\
\text { participants to } \\
\text { interventions }\end{array}$ & $\begin{array}{c}0 \\
(0.0)\end{array}$ & $\begin{array}{c}15 \\
(100)\end{array}$ & & \\
\hline
\end{tabular}

(continued) 


\begin{tabular}{|c|c|c|c|c|}
\hline Section/topic & No & Checklist item & $1^{\mathrm{a}}$ & $\mathbf{0}^{\mathbf{b}}$ \\
\hline \multirow[t]{2}{*}{ Blinding } & $11 \mathrm{a}$ & $\begin{array}{c}\text { If done, who } \\
\text { was blinded after } \\
\text { assignment to } \\
\text { interventions (for } \\
\text { example, participants, } \\
\text { care providers, those } \\
\text { assessing outcomes) } \\
\text { and how }\end{array}$ & $\begin{array}{c}2 \\
(13.3)\end{array}$ & $\begin{array}{c}13 \\
(86.7)\end{array}$ \\
\hline & $11 b$ & $\begin{array}{l}\text { If relevant, } \\
\text { description of } \\
\text { the similarity of } \\
\text { interventions }\end{array}$ & $\begin{array}{c}0 \\
(0.0)\end{array}$ & $\begin{array}{c}15 \\
(100)\end{array}$ \\
\hline \multirow{2}{*}{ Statistical methods } & $12 \mathrm{a}$ & $\begin{array}{l}\text { Statistical methods } \\
\text { used to compare } \\
\text { groups for primary } \\
\text { and secondary } \\
\text { outcomes }\end{array}$ & $9(60.0)$ & $6(40.0)$ \\
\hline & $12 b$ & $\begin{array}{c}\text { Methods for } \\
\text { additional analyses, } \\
\text { such as subgroup } \\
\text { analyses and adjusted } \\
\text { analyses }\end{array}$ & $\begin{array}{c}4 \\
(26.7)\end{array}$ & $\begin{array}{c}11 \\
(73.3)\end{array}$ \\
\hline
\end{tabular}

\section{Results}

Participant flow (a diagram is strongly recommended)

Recruitment

Baseline data

\begin{tabular}{|c|c|c|c|}
\hline $\begin{array}{l}\text { For each group, } \\
\text { the numbers of } \\
\text { participants who were } \\
\text { randomly assigned, } \\
\text { received intended } \\
\text { treatment, and were } \\
\text { analyzed for the } \\
\text { primary outcome }\end{array}$ & $\begin{array}{c}15 \\
(100)\end{array}$ & $\begin{array}{c}0 \\
(0.0)\end{array}$ & \\
\hline $\begin{array}{l}\text { For each group, } \\
\text { losses and exclusions } \\
\text { after randomization, } \\
\text { together with reasons }\end{array}$ & $\begin{array}{c}0 \\
(0.0)\end{array}$ & $\begin{array}{c}15 \\
(100)\end{array}$ & \\
\hline $\begin{array}{l}\text { Dates defining the } \\
\text { periods of recruitment } \\
\text { and follow-up }\end{array}$ & $\begin{array}{c}0 \\
(0.0)\end{array}$ & $\begin{array}{c}14 \\
(93.3)\end{array}$ & $\begin{array}{c}1 \\
(6.7)\end{array}$ \\
\hline $\begin{array}{c}\text { Why the trial ended } \\
\text { or was stopped }\end{array}$ & $\begin{array}{c}5 \\
(33.3)\end{array}$ & $\begin{array}{c}10 \\
(66.7)\end{array}$ & \\
\hline $\begin{array}{l}\text { A table showing } \\
\text { baseline demographic } \\
\text { and clinical } \\
\text { characteristics for } \\
\text { each group }\end{array}$ & $\begin{array}{c}11 \\
(73.3)\end{array}$ & $\begin{array}{c}3 \\
(20.0)\end{array}$ & $\begin{array}{c}1 \\
(6.7)\end{array}$ \\
\hline
\end{tabular}

(continued) 


\begin{tabular}{|c|c|c|c|c|c|c|}
\hline Section/topic & No & Checklist item & $1^{\mathrm{a}}$ & $\mathbf{0}^{\mathbf{b}}$ & $\mathrm{NI}^{\mathrm{c}}$ & Remark \\
\hline Numbers analyzed & 16 & $\begin{array}{c}\text { For each group, } \\
\text { number of } \\
\text { participants } \\
\text { (denominator) } \\
\text { included in each } \\
\text { analysis and whether } \\
\text { the analysis was by } \\
\text { original assigned } \\
\text { groups }\end{array}$ & $\begin{array}{c}15 \\
(100)\end{array}$ & $\begin{array}{c}0 \\
(0.0)\end{array}$ & & \\
\hline \multirow[t]{2}{*}{ Outcomes and estimation } & $17 \mathrm{a}$ & $\begin{array}{l}\text { For each primary and } \\
\text { secondary outcome, } \\
\text { results for each group, } \\
\text { and the estimated } \\
\text { effect size and its } \\
\text { precision (such as } \\
95 \% \text { confidence } \\
\text { interval) }\end{array}$ & $\begin{array}{c}13 \\
(86.7)\end{array}$ & $\begin{array}{c}0 \\
(0.0)\end{array}$ & $\begin{array}{c}2 \\
(13.3)\end{array}$ & \\
\hline & $17 \mathrm{~b}$ & $\begin{array}{l}\text { For binary outcomes, } \\
\text { presentation of } \\
\text { both absolute and } \\
\text { relative effect sizes is } \\
\text { recommended }\end{array}$ & $\begin{array}{c}0 \\
(0.0)\end{array}$ & $\begin{array}{c}15 \\
(100)\end{array}$ & & \\
\hline Ancillary analyses & 18 & $\begin{array}{l}\text { Results of any other } \\
\text { analyses performed, } \\
\text { including subgroup } \\
\text { analyses and } \\
\text { adjusted analyses, } \\
\text { distinguishing } \\
\text { pre-specified from } \\
\text { exploratory }\end{array}$ & $\begin{array}{c}0 \\
(0.0)\end{array}$ & $\begin{array}{c}15 \\
(100)\end{array}$ & & \\
\hline Harms & 19 & $\begin{array}{l}\text { Important harms or } \\
\text { unintended effects } \\
\text { in each group (for } \\
\text { specific guidance } \\
\text { see CONSORT for } \\
\text { harms) }\end{array}$ & $\begin{array}{c}14 \\
(93.3)\end{array}$ & $\begin{array}{c}1 \\
(6.7)\end{array}$ & & \\
\hline \multicolumn{7}{|l|}{ Discussion } \\
\hline Limitations & 20 & $\begin{array}{l}\text { Trial limitations, } \\
\text { addressing sources } \\
\text { of potential bias, } \\
\text { imprecision, and, if } \\
\text { relevant, multiplicity } \\
\text { of analyses }\end{array}$ & $6(40.0)$ & $9(60.0)$ & & \\
\hline Generalizability & 21 & $\begin{array}{l}\text { Generalizability } \\
\text { (external validity, } \\
\text { applicability) of the } \\
\text { trial findings }\end{array}$ & $\begin{array}{c}13 \\
(86.7)\end{array}$ & $\begin{array}{c}2 \\
(13.3)\end{array}$ & & \\
\hline
\end{tabular}

(continued) 


\begin{tabular}{|c|c|c|c|c|c|c|}
\hline Section/topic & No & Checklist item & $1^{a}$ & $\mathbf{0}^{\mathrm{b}}$ & $\mathrm{NI}^{\mathrm{c}}$ & Remark \\
\hline Interpretation & 22 & $\begin{array}{l}\text { Interpretation } \\
\text { consistent with } \\
\text { results, balancing } \\
\text { benefits and harms, } \\
\text { and considering other } \\
\text { relevant evidence }\end{array}$ & $7(46.7)$ & $4(26.7)$ & $4(26.7)$ & \\
\hline \multicolumn{7}{|c|}{ Other information } \\
\hline Registration & 23 & $\begin{array}{l}\text { Registration number } \\
\text { and name of trial } \\
\text { registry }\end{array}$ & $\begin{array}{c}0 \\
(0.0)\end{array}$ & $\begin{array}{c}15 \\
(100)\end{array}$ & & \\
\hline Protocol & 24 & $\begin{array}{l}\text { Where the full trial } \\
\text { protocol can be } \\
\text { accessed, if available }\end{array}$ & $\begin{array}{c}0 \\
(0.0)\end{array}$ & $\begin{array}{c}15 \\
(100)\end{array}$ & & \\
\hline Funding & 25 & $\begin{array}{l}\text { Sources of funding } \\
\text { and other support } \\
\text { (such as supply of } \\
\text { drugs), role of funders }\end{array}$ & $\begin{array}{c}3 \\
(20.0)\end{array}$ & $\begin{array}{c}12 \\
(80.0)\end{array}$ & & \\
\hline
\end{tabular}

Notes: check measure as $1^{\mathrm{a}}$ : reported; $0^{\mathrm{b}}$ : not reported; NIc: partially reported, but insufficient. STRICTA: Standards for Reporting Interventions in Clinical Trials of Acupuncture. RCTs: Randomized controlled trials.

increasing attention [27-30]. However chemotherapyincluded hematologic toxicity still exists. It not only increases pain to the patient, but also reduce medication adherence, delay the treatment cycle, and even lead to life-threatening events. Thus it is important for the patient to control hematologic toxicity after treatment with chemotherapy.

Over the past decade, many types of research focused on the relationship between cytokines and chemotherapy-included hematologic toxicity [31-36].

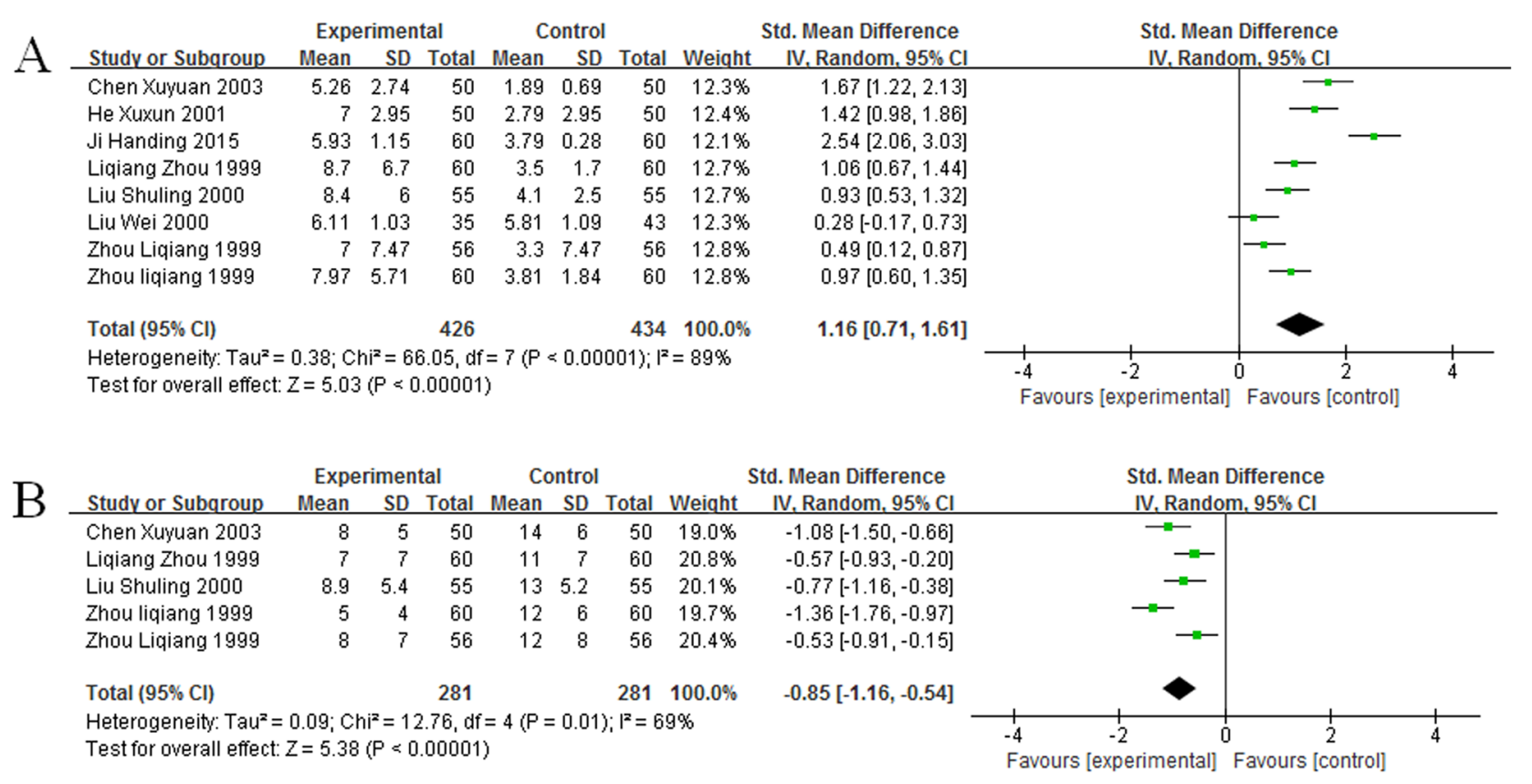

Figure 2: Forest plot showing the connection between GM-CSF and WBC count. (A) Impact of GM-CSF on WBC count. (B) Impact of GM-CSF on the recovery time of leukopenia. 
Since GM-CSF and granulocyte colony stimulating factor (G-CSF) were approved by FDA in the 1990s, they were both widely used to treat various types of cancer patients with chemotherapy-induced neutropenia and leucopenia, and achieved an excellent result. Because of the outstanding clinical efficacy of GM-CSF and G-CSF, they have made hundreds of millions of dollars each year in sales. G-CSF, as a member of cytokine families, is often used to promote the production of granulocytes or antigen presenting cells. And the clinical efficacy of G-CSF has been systematically evaluated and analyzed in many studies [10, 37-39]. However, as a cytokine that has a similar efficacy to G-CSF, the clinical efficacy of GMCSF is lack of systematic analysis and evaluation. Thus this study fills the gap. The result of our study confirmed that GM-CSF can help cancer patient after treatment of chemotherapy quickly improving WBC count and
ANC. And GM-CSF has no association with PLT count, however, the result regarding GM-CSF and PLT from A. Le Cesne et al. [16] were that GM-CSF could significantly improve the PLT count in cancer patients. Besides, GMCSF has a significant connection with fever.

Although the included data come from different countries, including Italia, Canada, France, America, and China. The drugs (GM-CSF) that they used were manufactured in different places and even have different trade names. The types of patients were all cancer patients after treatment of chemotherapy, administration route were all intravenous injection, administration time was all at least 24 hours after treatment of chemotherapy, and clinical observation index was all hematologic index. moreover, the clinical response rate in these studies was similar (73.7\%-97\%) except A. Le Cesne et al. [16] $(21.3 \%)$. Besides, three studies were published by one first

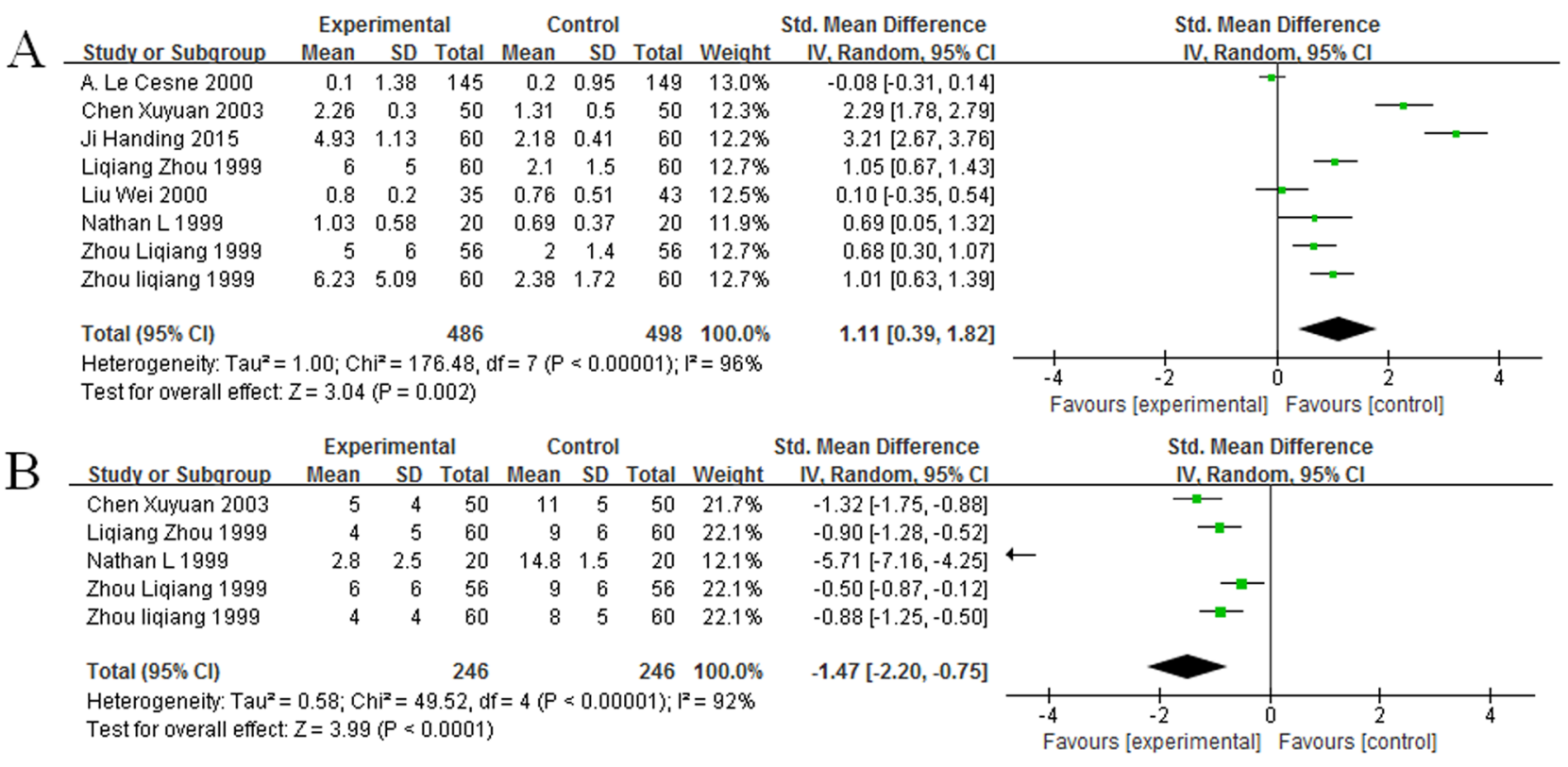

Figure 3: Forest plot showing the connection between GM-CSF and ANC. (A) Impact of GM-CSF on ANC count. (B) Impact of GM-CSF on the recovery time of neutropenia.

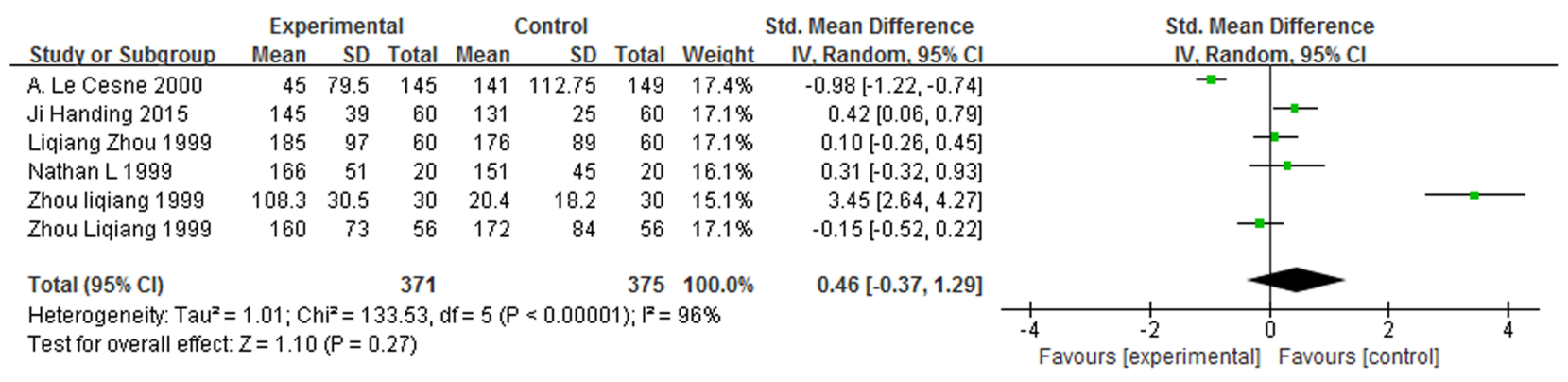

Figure 4: Forest plot showing the connection between GM-CSF and PLT count. 


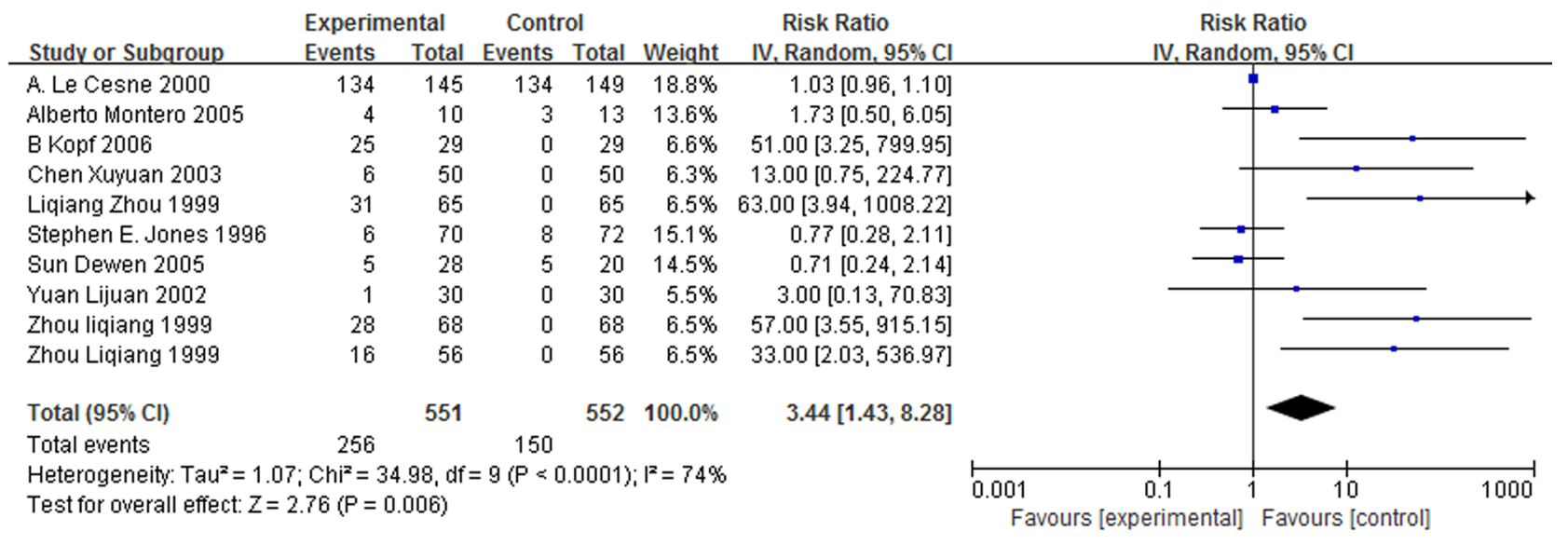

Figure 5: Forest plot showing the connection between GM-CSF and fever. Notes: Invalid lines vertical horizontal scale is 1. Each horizontal line represents the upper and lower bounds of the $95 \%$ confidence interval of the study. The length of the line represents the range of confidence interval. The green or blue solid in the center of a horizontal line is the positions of OR value or RR value, and the size of solid represents the weight of the study. Black diamond represents the effect quantity and confidence interval of multiple studies merging.
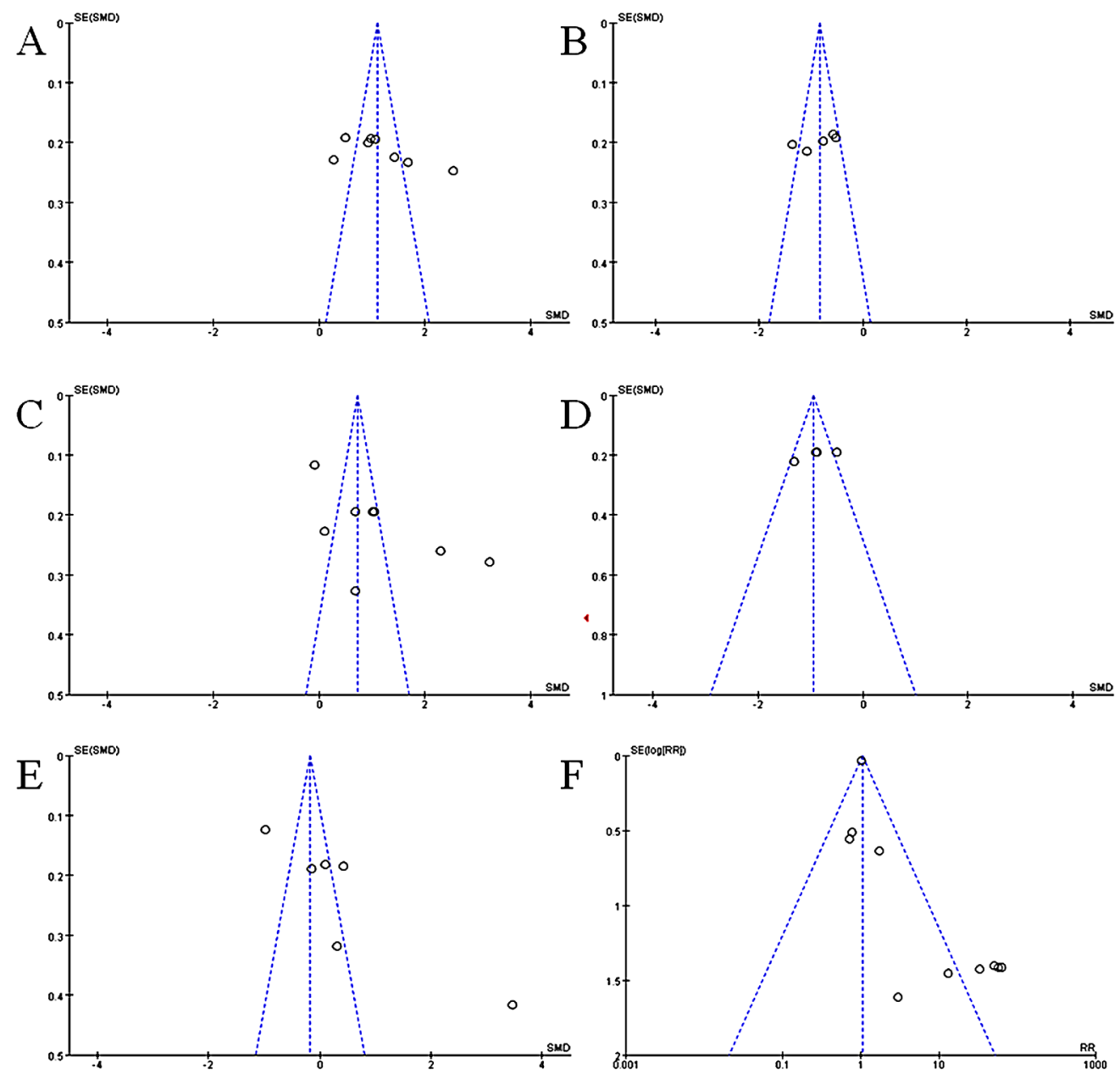

Figure 6: Funnel plot for publication bias. (A) Impact of GM-CSF on WBC count. (B) Impact of GM-CSF on the time of leukopenia. (C) Impact of GM-CSF on ANC. (D) Impact of GM-CSF on the time of neutropenia. (E) Impact of GM-CSF on PLT count. (F) Impact of GM-CSF on fever. 
author Zhou LQ [18, 23, 26], which means that Zhou was a specialist in this field. And these three studies used three different brands of GM-CSF to treat different patients at the same dosage and duration. It not only avoided the difference in the research system but also studied drug standardization of GM-CSF.

Our study also exists some limitations, the main limitations are sample size and the publication time of studies. According to the data needs of this study, the result of the search was so. The method and the duration of GMCSF administration still keep the same. The publication bias is not robust. And the data of statistical analysis were expressed in median and range. That was the reason why we did this study. We tried to find the connections between GM-CSF and hematologic index by analyzing available data, which can help the doctor better observe the efficacy of GM-CSF at the treatment stage, rather than waiting until the end of treatment.

The potential heterogeneity come from patients, there are over 30 cancer types with the total patients number, including lung cancer, breast cancer, lymphoma etc. And they were treated with different chemotherapy. However, firstly when GM-CSF is clinically used for chemotherapy-induced neutropenia and leucopenia, it is a broad-spectrum drug that does not target a particular tumor. GM-CSF is used simultaneously with chemotherapy drugs. The rapid proliferation and differentiation of myeloid precursor cells are sensitive to chemotherapeutic drugs, thus affecting the efficacy of chemotherapy. Secondly, the mechanism of GM-CSF therapy is actually immunotherapy. Thus the heterogeneity in immunity of patients is more important than cancer types. Unfortunately the data included in our metaanalysis lack the immunity information of patients.

In conclusion, according to the results of this study, we found that an included study [16] shown a significant difference from the other studies in two results: the impact of GM-CSF on PLT count and the clinical response rate of GM-CSF, respectively. The most likely reason for this is sources of medication, however, we cannot rule out other possible causes. Thus we believed that only the index of WBC count and ANC were used to judge the outcome of GM-CSF treatment in previous studies, which may not completely reflect the efficacy. We suggested that hematological indexes need to be expanded in future studies. Further, the other indexes should also be observed, such as immunological indexes.

\section{MATERIALS AND METHODS}

\section{Search strategy}

Firstly we searched the systematic reviews and meta-analysis on the role of GM-CSF in cancer patient after treatment of chemotherapy. No reviews or meta-analysis were found. Secondly, we searched the
PubMed and Wanfang databases for studies reporting the use of GM-CSF in cancer patient after treatment of chemotherapy. And the following keywords were used in the process of search, respectively: GM-CSF cancer therapy clinic, GM-CSF white blood cell, sargramostim, leucomax and molgramostim. The languages of articles were limited in English and Chinese. "Full text" and "Human" were used to filter article. Finally, the reference lists of primary studies were reviewed by two authors. The latest search happened on March 9, 2017.

\section{Selection criteria for considering studies for this review}

The patients from included studies were diagnosed with various forms of cancer, and all of them received systemic chemotherapy. At the same time, they agreed to participate in an experimental study of the impact of GM-CSF on hematologic indexes and complications. Then we excluded the following studies: (1) editorials, abstract and letters, (2) studies in which the clinical outcome was not reported, (3) single-patient case report or short case series involving fewer than four patients (4) multiple publications from the same study to prevent erroneous patient count and (5) graduation theses.

\section{Initial review of studies}

The initial database was compiled, and all duplicate articles were eliminated. We screened these citations depending on title, abstract and the relevant studies for inclusion based on the criteria identified previously. Only after assessment of the full-text articles by two authors, the studies were finally selected for inclusion in the review. Any disagreement was resolved by discussion between two authors.

\section{Data abstraction}

The data of initial review were recorded on a standard data extraction form by both the authors independently. The following items were extracted: (1) publication details (title, authors, years and other citation details, including geographic locale); (2) type of study (clinical trial or review); (3) treatment details, (chemotherapy or radiotherapy); (4) patients details (number, sex ratio, age, weight, KPS); (5) dosage and duration of GM-CSF; (6) sources of medication; and (7) clinical response and toxic or side effects, if any.

\section{Assessment of study quality}

The CONSORT 2010 checklist (25 items) was used to assess the reporting quality of eligible studies. According to this checklist, two authors assessed the reporting quality, respectively. Every 25 items had one common criterion, including "yes", "no" and "I". They 
represented "adequately reported", "Not reported" and "partially reported, but insufficient", respectively. Besides, "unable to judge" represented if there was lacking information regarding the item. Any disagreement was resolved by discussion between the two authors.

\section{Statistical analysis}

Cochran's $Q$ test and Higgins' $\mathrm{I}^{2}$ statistics were used to making homogeneity test for eligible studies. A $P$-value $\leq 0.1$ and/or $\mathrm{I}^{2} \geq 50 \%$ indicated significant heterogeneity, the data should be calculated by the random-effect model. Accordingly A $P$-value $>0.1$ and/or $\mathrm{I}^{2}<50 \%$ indicated significant homogeneity, the data should be calculated by fixed-effect model [40]. Standard mean differences (SMD) and pooled risk ratios (RR) with 95\% confidence intervals $(95 \% \mathrm{CI})$ were used to evaluate the association of GM-CSF with hematologic indexes and complications, respectively. The $\mathrm{Z}$ test was used in effect comparison between experimental and control. A $P$-value $<0.05$ in the $\mathrm{Z}$ test for pooled RR, or no overlap of the $95 \% \mathrm{CI}$ with 1 was considered statistically significant. And the publication bias was assessed by visual inspection of funnel plots. All data analyses were performed by using Review Manager 5.3 (Cochrane Collaboration, London, UK) and adhered to the PRISMA guidelines.

\section{CONFLICTS OF INTEREST}

All the authors declare to have no conflicts of interest.

\section{FUNDING}

This study was supported by grants from the National Key R\&D Program by of China (2016YFC0902702, 2017YFA0104301, 2017YFA0506002), the Chinese National Natural Sciences Foundation (81630092, 81421091).

\section{REFERENCES}

1. Rohatgi S, Jagannathan JP, Rosenthal MH, Kim KW, Ramaiya NH, Krajewski KM. Vascular toxicity associated with chemotherapy and molecular targeted therapy: what should a radiologist know? AJR Am J Roentgenol. 2014; 203:1353-62.

2. Magge RS, DeAngelis LM. The double-edged sword: neurotoxicity of chemotherapy. Blood Rev. 2015; 29:93-100.

3. Ratti M, Tomasello G. Lipegfilgrastim for the prophylaxis and treatment of chemotherapy-induced neutropenia. Expert Rev Clin Pharmacol. 2015; 8:15-24.

4. Cui R, Chu L, Liu ZQ, Xiao YY, Zhu XL, Chen YJ, Xu Q. Hematologic toxicity assessment in solid tumor patients treated with cetuximab: a pooled analysis of 18 randomized controlled trials. Int J Cancer. 2015; 136:936-44.

5. Miller RC, Steinbach A. Growth factor use in medicationinduced hematologic toxicity. J Pharm Pract. 2014; 27:453-460.

6. Shitara K, Matsuo K, Oze I, Mizota A, Kondo C, Nomura M, Yokota T, Takahari D, Ura T, Muro K. Meta-analysis of neutropenia or leukopenia as a prognostic factor in patients with malignant disease undergoing chemotherapy. Cancer Chemother Pharmacol. 2011; 68:301-307.

7. Dreicer R, Klein EA, Elson P, Peereboom D, Byzova T, Plow EF. Phase II trial of GM-CSF+ thalidomide in patients with androgen-independent metastatic prostate cancer. Urol Oncol. 2005; 23:82-6.

8. Francisco-Cruz A, Aguilar-Santelises M, Ramos-Espinosa O, Mata-Espinosa D, Marquina-Castillo B, Barrios-Payan J, Hernandez-Pando R. Granulocyte-macrophage colonystimulating factor: not just another hematopoietic growth factor. Med Oncol. 2014; 31:774.

9. Däbritz J. Granulocyte macrophage colony-stimulating factor and the intestinal innate immune cell homeostasis in Crohn's disease. Am J Physiol Gastrointest Liver Physiol. 2014; 306:G455-65.

10. Mehta HM, Malandra M, Corey SJ. G-CSF and GM-CSF in neutropenia. J Immunol. 2015; 195:1341-9.

11. van Nieuwenhuijze A, Koenders M, Roeleveld D, Sleeman MA, van den Berg W, Wicks IP. GM-CSF as a therapeutic target in inflammatory diseases. Mol Immunol. 2013; 56:675-682.

12. Kopf B, De Giorgi U, Vertogen B, Monti G, Molinari A, Turci D, Dazzi C, Leoni M, Tienghi A, Cariello A. A randomized study comparing filgrastim versus lenograstim versus molgramostim plus chemotherapy for peripheral blood progenitor cell mobilization. Bone Marrow Transplant. 2006; 38:407-12.

13. Kobrinsky NL, Sjolander DE, Cheang MS, Levitt R, Steen PD. Granulocyte-macrophage colony-stimulating factor treatment before doxorubicin and cyclophosphamide chemotherapy priming in women with early-stage breast cancer. J Clin Oncol. 1999; 17:3426-30.

14. Montero A, Booser D, Esparza-Guerra L, Murray J, Rosales M, Hortobagyi G, Valero V. Phase I trial of dose-dense docetaxel and doxorubicin with or without sargramostim in patients with metastatic breast cancer. Drugs R D. 2005; 6:299-305.

15. Jones SE, Schottstaedt MW, Duncan LA, Kirby RL, Good RH, Mennel RG, George TK, Snyder DA, Watkins DL, Denham CA. Randomized double-blind prospective trial to evaluate the effects of sargramostim versus placebo in a moderate-dose fluorouracil, doxorubicin, and cyclophosphamide adjuvant chemotherapy program for stage II and III breast cancer. J Clin Oncol. 1996; 14:2976-83.

16. Le Cesne A, Judson I, Crowther D, Rodenhuis S, Keizer H, Van Hoesel Q, Blay JY, Frisch J, Van Glabbeke M, Hermans C, Van 
Oosterom A, Tursz T, Verweij J. Randomized phase III study comparing conventional-dose doxorubicin plus ifosfamide versus high-dose doxorubicin plus ifosfamide plus recombinant human granulocyte-macrophage colony-stimulating factor in advanced soft tissue sarcomas: A trial of the European Organization for Research and Treatment of Cancer/Soft Tissue and Bone Sarcoma Group. J Clin Oncol. 2000; 18:2676-84.

17. He XX, Xia XQ. Clinical observation of low doses of domestic GM-CSF on leukopenia after chemotherapy. Linchuang Huicui. 2001; 16:1.

18. Zhou LQ, Feng FY, Mo XN. Randomized controlled study of Gening (Domestic recombinant human granulocytemacrophage colony stimulating factor, rhGM-CSF) on leukopenia induced by combination chemotherapy. Cancer Res Prev Treat. 1999; 26.

19. Sun DW. Effect observation of 28 cases for Gening in treating chemotherapy-induced neutropenia. Chin Med J Commun. 2005; 19:2.

20. Liu SL, Wang Y, Chi CP, Li JP, Liu DM, Zhang XX, Jing D, Zhang CL, Ji HB, Yang GY, Song SQ, Jiang ZF, Liu CF, et al. Phase II clinical effect observation of domestic rhGMCSF. Chin J Biol. 2000; 13:3.

21. Chen XY, Zhu YX, Chen XP, Dai XB, Zhang L, Lei ZZ. Clinical research of Liyaer prevention of chemotherapyinduced neutropenia. Chongoqing Med J. 2003; 32:2.

22. Liu W, Feng WJ, Hu SJ. Collaborative therapy with chemotherapy plus Libaiduo for advanced cancer in 78 cases. J Hebei Med Univ. 2001; 22:2.

23. Zhou LQ, Feng FY, Zhou SY. Randomized contrastive phase II clinical study of Leiboitai preventing leukopenia induced by tumor chemotherapy. Chin J Clin Oncol. 1999; 26:1.

24. Ji HD. Effect observation of external use rhGM-CSF to treat common disease complications of tumer. Yiayao Qianyan. $2015 ; 5: 2$.

25. Yuan LJ, Zhang HW. Clinical study of recombinant human granulocyte colony-stimulating factor hemoglobin in leukopenia. Med J Commun. 2002; 16:2.

26. Zhou LQ, Zhou SY, Feng FY. Recombinant human granulocyte macrophage colony-stimulating factor on leucopenia patients phase II clinical trials. Chin J New Drug Clin Remedies. 1999; 18.

27. Perez-Herrero E, Fernandez-Medarde A. Advanced targeted therapies in cancer: drug nanocarriers, the future of chemotherapy. Eur J Pharm Biopharm. 2015; 93:52-79.

28. Li CJ, Liu XZ, Zhang L, Chen LB, Shi X, Wu SJ, Zhao $J \mathrm{~N}$. Advances in bone-targeted drug delivery systems for neoadjuvant chemotherapy for osteosarcoma. Orthop Surg. 2016; 8:105-10.
29. Kuo HY, Yeh KH. Molecular-targeted therapy for chemotherapy-refractory gastric cancer: a case report and literature review. Anticancer Res. 2014; 34:3695-9.

30. Diamond E, Molina AM, Carbonaro M, Akhtar NH, Giannakakou P, Tagawa ST, Nanus DM. Cytotoxic chemotherapy in the treatment of advanced renal cell carcinoma in the era of targeted therapy. Crit Rev Oncol Hematol. 2015; 96:518-26.

31. Bennett JM, Young MS, Liesveld JL, Paietta E, Miller KB, Lazarus HM, Marsh RD, Friedenberg WR, Saba HT, Hayes FA, Dewald GW, Hiddemann W, Rowe JM. Phase II study of combination human recombinant GM-CSF with intermediatedose cytarabine and mitoxantrone chemotherapy in patients with high-risk myelodysplastic syndromes (RAEB, RAEBT, and CMML): an Eastern Cooperative Oncology Group Study. Am J Hematol. 2001; 66:23-7.

32. Armitage JO. Emerging applications of recombinant human granulocyte-macrophage colony-stimulating factor. Blood. 1998; 92:4491-508.

33. Fruehauf JP, Kong KM, Jakowatz JG. Docetaxel and vinorelbine plus GM-CSF in malignant melanoma. Oncology (Williston Park). 2005; 19:19-22.

34. Winer ES, Miller KB, Chan GW. GM-CSF and low-dose cytosine arabinoside in high-risk, elderly patients with AML or MDS. Oncology. 2005; 19:11-4.

35. Thomas X. New emerging applications of molgramostim in acute myeloid leukaemia. Expert Opin Drug Metab Toxicol. 2008; 4:795-806.

36. Balducci L, Carreca I. The role of myelopoietic growth factors in managing cancer in the elderly. Drugs. 2002; 62:47-63.

37. Disis ML. Clinical use of subcutaneous G-CSF or GM-CSF in malignancy. Oncology. 2005; 19:5-9.

38. Quittet P, Ceballos P, Lopez E, Lu ZY, Latry P, Becht C, Legouffe E, Fegueux N, Exbrayat C, Pouessel D, Rouille V, Daures JP, Klein B, et al. Low doses of GM-CSF (molgramostim) and G-CSF (filgrastim) after cyclophosphamide $(4 \mathrm{~g} / \mathrm{m} 2)$ enhance the peripheral blood progenitor cell harvest: results of two randomized studies including 120 patients. Bone Marrow Transplant. 2006; 38:275-84.

39. Weaver CH, Schulman KA, Buckner CD. Mobilization of peripheral blood stem cells following myelosuppressive chemotherapy: a randomized comparison of filgrastim, sargramostim, or sequential sargramostim and filgrastim. Bone Marrow Transplant. 2001; 27:S23-9.

40. Moore RA, Edwards JE, McQuay HJ. Acute pain: individual patient meta-analysis shows the impact of different ways of analyzing and presenting results. Pain. 2005; 116:322-31. 